\title{
Pathway-based analysis of a genome-wide case-control association study of rheumatoid arthritis

\author{
Joseph Beyene ${ }^{* 1,2,3}$, Pingzhao $\mathrm{Hu}^{3}$, Jemila S Hamid ${ }^{1}$, Elena Parkhomenko ${ }^{1}$, \\ Andrew D Paterson ${ }^{2,3}$ and David Tritchler ${ }^{2,4,5}$
}

\begin{abstract}
Addresses: ${ }^{1}$ Biostatistics Methodology Unit, Research Institute, Hospital for Sick Children, 555 University Avenue, Toronto, Ontario M5G 1X8, Canada, ${ }^{2}$ Dalla Lana School of Public Health, University of Toronto, Health Sciences Building, 155 College Street, Toronto, Ontario M5T 3M7, Canada, ${ }^{3}$ The Centre for Applied Genomics, The Hospital for Sick Children Research Institute, 101 College Street, Toronto, Ontario M5G 1L7, Canada, ${ }^{4}$ Division of Epidemiology and Statistics, Ontario Cancer Institute, 610 University Avenue, Toronto, Ontario M5G 2M9, Canada and ${ }^{5}$ Department of Biostatistics, State University of New York at Buffalo, 249 Farber Hall, 3435 Main Street, Building 26, Buffalo, New York 14214-3000, USA
\end{abstract}

E-mail: Joseph Beyene* - joseph@utstat.toronto.edu; Pingzhao Hu - phu@sickkids.ca; Jemila S Hamid - jemila@utstat.toronto.edu; Elena Parkhomenko - elena@utstat.utoronto.ca; Andrew D Paterson - andrew.paterson@utoronto.ca;

David Tritchler - tritchle@uhnres.utoronto.ca

*Corresponding author

from Genetic Analysis Workshop 16

St Louis, MO, USA 17-20 September 2009

Published: 15 December 2009

BMC Proceedings 2009, 3(Suppl 7):SI28 doi: 10.1186/1753-656I-3-S7-SI28

This article is available from: http://www.biomedcentral.com/I753-656I/3/S7/SI28

(C) 2009 Beyene et al; licensee BioMed Central Ltd.

This is an open access article distributed under the terms of the Creative Commons Attribution License (http://creativecommons.org/licenses/by/2.0), which permits unrestricted use, distribution, and reproduction in any medium, provided the original work is properly cited.

\begin{abstract}
Evaluation of the association between single-nucleotide polymorphisms (SNPs) and disease outcomes is widely used to identify genetic risk factors for complex diseases. Although this analysis paradigm has made significant progress in many genetic studies, many challenges remain, such as the requirement of a large sample size to achieve adequate power. Here we use rheumatoid arthritis (RA) as an example and explore a new analysis strategy: pathway-based analysis to search for related genes and SNPs contributing to the disease.

We first propose the application of measure of explained variation to quantify the predictive ability of a given SNP. We then use gene set enrichment analysis to evaluate enrichment of specific pathways, where pathways, are considered enriched if they consist of genes that are associated with the phenotype of interest above and beyond is expected by chance. The results are also compared with score tests for association analysis by adjusting for population stratification.

Our study identified some significantly enriched pathways, such as "cell adhesion molecules," which are known to play a key role in RA. Our results showed that pathway-based analysis may identify other biologically interesting loci (e.g., rs I0I836I) related to RA: the gene (CTLA4) closest to this marker has previously been shown to be associated with RA and the gene is in the significant pathways we identified, even though the marker has not reached genome-wide significance in univariate single-marker analysis.
\end{abstract}




\section{Background}

Rheumatoid arthritis (RA) is the most common systemic autoimmune disease characterized by chronic, destructive, and debilitating inflammation of joints and extraarticular tissues [1]. The disease affects $1 \%$ of the adult population worldwide and is significantly more prevalent in women ( 3 to 1 ratio) than men. It is believed that the contribution of individual genes to complex diseases such as RA, is generally modest and difficult to detect due to inadequate sample sizes compared with the large number of variables being tested for association. Currently, the literature on genome-wide association studies is focused on testing single-nucleotide polymorphisms (SNPs) for association using standard test statistic (for example, chi-square test statistic). The difficulty in detecting genes of modest effects may be one potential explanation for inconsistent results often seen in genetic association studies [2]. In a recent genome-wide study of RA with a relatively large sample size, Plenge et al. [3] identified a genome-wide significant association signal on chromosome 9 close to TRAF1 and C5 in addition to confirming known genes related to RA (e.g., HLA-DRB1 in major histocompatibility complex (MHC) region and PTPN22) [3].

In this paper, we propose a pathway-based approach to study sets of genes. The motivation for this is the belief that the mechanism of a complex disease such as RA may not be described fully by looking at gene-by-gene comparisons alone. Gene set analysis has been widely used in studies involving gene expression data; however, there are only a few such studies in a genome-wide association setting. We also propose two measures of explained variation that are appropriate for binary outcomes and summarize these measurements over all SNPs in and around a particular gene.

\section{Materials and methods Data description}

The provided data is a subset of the Stage 1 genome-wide association study of RA previously analyzed by Plenge et al. [3]. After removing duplicated and contaminated samples, there were 868 cases from the North American Rheumatoid Arthritis Consortium (NARAC) and 1194 controls on which 545,080 SNPs had been genotyped.

\section{Quality control}

The SNPs and samples fulfilling the following quality control requirements were included in our analysis: 1) call rate: SNP and sample call rates > 95\%; 2) HardyWeinberg equilibrium: false-discovery rate (FDR) level in testing for Hardy-Weinberg equilibrium among controls $>0.2$; 3) minor allele count $>10$ copies, which is equivalent to a minor allele frequency of $0.24 \%$ (i.e, $10 /$
$(2 \times 2062)=0.24 \%)$. A total of 490,000 SNPs and 2062 samples met our quality control criteria and were used for further analysis.

\section{Adjustment for population stratification}

It has been indicated that this data set is affected by population stratification, and this may lead to misleading association results if not taken into account. We followed an approach proposed by Price et al. [4] to adjust for population stratification. The steps involved can be summarized as follows: i) Using the SNP set remaining after applying our quality control criteria, we first removed SNPs in the MHC region (29-34 Mb on chromosome 6) and on the sex chromosomes. ii) For the remaining SNPs, we pruned them based on linkage disequilibrium using PLINK [5]. iii) This left around 220 $\mathrm{k}$ SNPs for which we calculated genome-wide pair-wise identity-by-state similarity matrix followed by multidimensional scaling analysis on the identity-by-statebased distance matrix. iv) We selected 15 significant principal coordinates (PCs) with $p$-value $<0.001$. v) These 15 significant PCs were used in subsequent association analyses. It should be noted that these steps were only applied to select these 15 significant PCs. In the subsequent association analyses, we used all SNPs and samples that passed the quality control criteria.

\section{Univariate SNP association test}

We performed score tests for association between RA status in patients and their genotypes, and adjusted for possible population stratification using the significant principal components. We used the 'egscore' function in GenABEL $R$ package [6], in which the population stratification method proposed by Price et al. [4] is implemented. We obtained the corresponding $p$-value and chi-square value for each SNP.

\section{Measures of explained variation}

For a given SNP, let $\left(y_{i}, x_{i}\right), i=1, \ldots, n$ denote its $n$ samples/observations, where $y_{i}$ denotes the outcome of the observation $i$ (in this study, $y_{i}=1$ if the subject is a case and 0 otherwise) and $x_{i}$ denotes the genotype (coded as $0,1,2$, corresponding to $A A, A B$, and $B B$ ) of the $i^{\text {th }}$ observation. We consider two measures of explained variation for binary outcomes [7]. These measures are based on direct and indirect estimates of predictive accuracy. The direct measure is based on residual from the fit and the indirect index is related to a standard measure of information. Let the estimates from a logistic model without a covariate be $\operatorname{Pr}\left\{y_{i}=1\right\} \equiv \bar{p}=\frac{\sum \gamma_{i}}{n}=\bar{\gamma}$ and the estimates from a model with covariate $x_{i}$ be $\operatorname{Pr}\left\{y_{i}=1 \mid x_{i}\right\}=\hat{p}_{i}$. Define 
$\hat{D}_{x}=n^{-1} \sum\left|y_{i}-\hat{p}_{i}\right|$ and $\hat{D}_{x}=n^{-1} \sum\left|y_{i}-\hat{p}_{i}\right|$, and the explained variation based on the direct estimates becomes $E V_{\text {direct }}=\left(\hat{D}-\hat{D}_{x}\right) / \hat{D} \times 100 \%$. Similarly, let $\tilde{D}=2 \bar{p}(1-\bar{p})$ and $\tilde{D}_{x}=2 n^{-1} \sum \hat{p}_{i}\left(1-\hat{p}_{i}\right)$, then the explained variation based on indirect estimates is calculated as $E V_{\text {indirect }}=\left(\tilde{D}-\tilde{D}_{x}\right) / \tilde{D} \times 100 \%$. We denote these two approaches as DirEV and IndirEV, respectively. In the models which include principal components to adjust for population stratification, the explained variation attributable to each SNP were obtained.

\section{Pathway-based analysis}

We summarize the steps for our method as follows (adapted from Wang et al. [8]):

\section{Obtain test/summary statistic}

For each SNP, we computed one of the following: measure of explained variation and univariate SNP association test $\left(\chi^{2}\right)$ where population stratification is adjusted for by including 15 PCs.

\section{Map SNPs to genes}

We obtained the nearest gene name for each SNP from the Illumina SNP annotation file (HumanHap650Yv3 Gene_Annotation.txt, available from https://icom. illumina.com/) based on physical distance. The 490,000 SNPs were mapped to 16,500 genes.

\section{Aggregate test/summary statistic}

For each gene, we obtained an aggregate summary measure or test statistic based on individual values (from Step 1 above) for SNPs assigned to this gene (Step 2). Here we used the maximum summary measure or maximum test statistic over all SNPs mapped to the gene.

4. The aggregated summary measure or test statistics were used to evaluate the significance of predefined gene sets/pathways [9] based on the gene set enrichment analysis (GSEA) method [10]. Here we used the c2 curated gene sets, which are obtained from online pathway databases, citation in PubMed [9], and knowledge of domain experts and included 1900 gene sets collected from canonical pathways, chemical and genetic perturbations, BioCarta pathways, GeneMAPP [11], and KEGG [12]. A Kolmogorov-Smirnov non-parametric rank statistic was performed using the GseaPreranked tool included in the GSEA software. Gene sets were ranked by their FDR $q$-value, where the $q$-value of a test measures the proportion of false positives incurred (FDR) when that particular test is called significant. The empirical null distribution was obtained using 1000 random permutations. We defined a given gene set as significantly enriched in the data if it has FDR $q$-value of less than 0.05 .

\section{Results}

For the 1900 gene sets that we evaluated, all three methods (Chi-Sq, DirEV and IndirEV) identified 10 gene sets that have FDR $q$-value of less than 0.05 . Table 1 shows these ten significantly enriched gene sets/pathways identified by GSEA for each of the three methods. As seen from Table 1, the ten significantly enriched pathways for the three summary methods are the same. The pathways identified using DirEv and IndirEV have similar FDR $q$-value and ranks. The FDR $q$-value results based on the $\chi^{2}$ tests are slightly different from those based on DirEV and IndirEV.

As discussed before, there are few potential genes associated with RA: PTPN22 on chromosome 1, HLA_DRB1 on the MHC region on chromosome 6, and TRAF1/C5 on chromosome 9. However, PTPN22 and TRAF1/C5 were not in any of the ten significantly enriched gene sets. Therefore, we further evaluated the distributions of genes and SNPs in the MHC region compared with the rest of the genome (those not in $\mathrm{MHC}$ region) in these ten gene sets/pathways (Table 2). To do this, we defined the $\mathrm{MHC}$ region as 29-34 Mb on chromosome 6 . The region is defined based on the results shown in Supplementary Table $1 \mathrm{~B}$ of Plenge et al. [3], that is, SNPs that have $p$-value $<0.0001$. We found 561 SNPs in the defined MHC region with $p$-value $<0.0001$; and 227 genes in the region using BioMart [13].

Table 2 shows the distribution of these genes and SNPs in the defined MHC region and other genes and SNPs with $p$-value $<0.0001$ in the rest of the genome in the 10 gene sets/pathways. It can be seen the MHC region is enriched for genes from the following three gene sets: "Hsa04612 Antigen Processing and Presentation," "Hsa04940 Type I Diabetes Mellitus," and "Hsa04514 Cell Adhesion Molecule." There were 22 HLA-related genes in common among these three gene sets. Of these, 20 were found in the defined MHC region. Five SNPs with $p$-value $<0.0001$ were present in these three gene sets in the MHC region. Interestingly, one SNP (rs1018361) with $p$-value of $2.6 \times 10^{-5}$ on chromosome 2 was present in one of the three gene sets ("Hsa04514 Cell Adhesion Molecule") in the rest of genome (the region excluding $\mathrm{MHC}$ region) while the other two gene sets had no significant SNPs in the rest of the genome.

The significant SNP on chromosome 2 (rs1018361) may be of interest for further investigation because the closest gene (CTLA4) to this SNP shares the same pathway as those genes containing the MHC region. Moreover, 
Table I: Significant pathways identified by three test statistic methods

\begin{tabular}{|c|c|c|c|c|c|c|c|}
\hline \multirow[b]{2}{*}{ Gene sets/pathways } & \multirow[b]{2}{*}{ No. genes ${ }^{a}$} & \multicolumn{2}{|r|}{$\chi^{2}$} & \multicolumn{2}{|c|}{ DirEV } & \multicolumn{2}{|c|}{ IndirEV } \\
\hline & & Rank $^{\mathbf{b}}$ & FDR q-value & Rank & FDR q-value & Rank & FDR $q$-value \\
\hline Hsa046I2 antigen processing and presentation & 57 & 1 & $<1 * 10^{-8}$ & I & $<1 * 10^{-8}$ & I & $<1 * 10^{-8}$ \\
\hline Hsa04940 Type I diabetes mellitus & 40 & 2 & $<1 * 10^{-8}$ & 2 & $<1 * 10^{-8}$ & 2 & $<1 * 10^{-8}$ \\
\hline Wieland hepatitis B-induced & 87 & 3 & $<1 * 10^{-8}$ & 3 & 0.004 & 3 & 0.001 \\
\hline Ctla4 pathway & 18 & 4 & $6 * 10^{-5}$ & 6 & 0.006 & 4 & 0.003 \\
\hline Ami pathway & 22 & 5 & 0.01 & 7 & 0.006 & 10 & 0.015 \\
\hline Csk pathway & 22 & 6 & 0.01 & 10 & 0.011 & 7 & 0.012 \\
\hline Sana Ifng endothelial up & 67 & 7 & 0.011 & 8 & 0.006 & 9 & 0.013 \\
\hline Th1 th2 pathway & 17 & 8 & 0.011 & 9 & 0.009 & 8 & 0.013 \\
\hline Inflam pathway & 28 & 9 & 0.012 & 4 & 0.005 & 6 & 0.006 \\
\hline Hsa045I 4 cell adhesion molecule & 115 & 10 & 0.02 & 5 & 0.005 & 5 & 0.004 \\
\hline
\end{tabular}

${ }^{a}$ Number of genes found in our data. (The actual number of genes defined in the set is larger than these numbers.)

${ }^{\mathrm{b}}$ The rank is based on the FDR $q$-value for all I,900 tested gene sets in each method.

Table 2: Distribution of selected genes and SNPs in each of the two regions and nine gene sets/pathways

\begin{tabular}{|c|c|c|c|c|c|c|}
\hline \multirow[b]{2}{*}{ Gene sets/pathways } & \multicolumn{3}{|c|}{$\begin{array}{c}\text { MHC region } \\
\left(\mathrm{Chr} 6,29-34 \mathrm{Mb}^{\mathrm{a}}\right)\end{array}$} & \multicolumn{3}{|c|}{ Rest of genome } \\
\hline & No. genes & $\%^{\mathbf{b}}$ & \#SNPs & No. genes & $\%^{\mathbf{a}}$ & No. SNPs \\
\hline Hsa046I2 antigen processing and Presentation & 24 & 42.1 & 5 & 33 & 57.9 & 0 \\
\hline Hsa04940 Type I Diabetes Mellitus & 22 & 55.0 & 6 & 18 & 45.0 & 0 \\
\hline Wieland hepatitis B-ilnduced & 15 & 17.2 & 4 & 72 & 82.8 & 0 \\
\hline Ctla4 pathway & 2 & I I.I & 0 & 16 & 88.9 & 1 \\
\hline Ami pathway & 2 & 9.1 & 0 & 20 & 90.9 & 0 \\
\hline Csk pathway & 2 & 9.1 & 0 & 20 & 90.9 & 0 \\
\hline Sana Ifng endothelial up & II & 16.4 & 2 & 56 & 83.6 & 0 \\
\hline ThI th2 pathway & 2 & 11.8 & 0 & 15 & 88.2 & 0 \\
\hline Inflam pathway & 4 & 14.3 & 2 & 24 & 85.7 & 0 \\
\hline Hsa045I 4 cell adhesion molecule & 20 & 17.4 & 5 & 95 & 82.6 & 1 \\
\hline
\end{tabular}

${ }^{a}$ This region covers approximately $0.2 \%$ the whole genome

b The percentage of genes in a given gene set that were found in the region

previous association study showed that RA is associated with CTLA4 [2]. Similarly, the cell adhesion molecules (proteins) may have an important role in regulation of the RA development than other tested pathways. As shown in Table 2, both genes and SNPs with $p$-value $<0.0001$ in the pathway (cell adhesion molecule) are found in both the MHC region and the rest of the genome, while other significant pathways have SNPs with $p$-value < 0.0001 only in the $\mathrm{MHC}$ region or are not found in either the $\mathrm{MHC}$ region or the rest of the genome.

\section{Conclusion}

Overall, using a pathway-based analysis, we found some of the significant gene sets/pathways were enriched in the well known MHC region associated with RA. However, some of the loci that have been reported to be related to RA, such as PTPN22 and TRAF1/C5, were not found to be enriched in any of the gene sets/ pathways we identified as significant. Our results also showed that pathway-based analysis may identify other RA-related loci (e.g., rs1018361) because the gene
(CTLA4) closest to this SNP has previously been shown to be associated with RA and the significant pathways identified here contained this gene.

\section{List of abbreviations used}

DirEV: Explained variation based on the direct estimates; FDR: False-discovery rate; GSEA: Gene set enrichment analysis; IndirEV: Explained variation based on indirect estimates; MHC: Major histocompatability complex; PC: Principal coordinates; RA: Rheumatoid arthritis; SNP: Single-nucleotide polymorphism.

\section{Competing interests}

The authors declare that they have no competing interests.

\section{Authors' contributions}

JB initiated the study and proposed the pathway-based analysis ideas and methods for genetic association analysis. $\mathrm{PH}$ performed all of the data analysis and drafted the paper with JB, JSH, and ADP. JSH, ADP, EP, 
and DT contributed by providing critical comments which helped with refining the methods, analyses, and interpretation of the data. All authors read and approved the final manuscript.

\section{Acknowledgements}

This work was partially supported by grants from the Natural Sciences and Engineering Research Council of Canada (NSERC), the Mathematics of Information Technology and Complex Systems (MITACS), Canadian Institute of Health Research (CIHR, grant 84392), and Genome Canada through the Ontario Genomics Institute. The Genetic Analysis Workshops are supported by NIH grant ROI GM03I575 from the National Institute of General Medical Sciences.

This article has been published as part of BMC Proceedings Volume 3 Supplement 7, 2009: Genetic Analysis Workshop 16. The full contents of the supplement are available online at http://www.biomedcentral.com/ $1753-6561 / 3$ ? issue $=$ S7.

\section{References}

I. Chang M, Rowland CM, Garcia VE, Schrodi SJ, Catanese J], Helm-van Mil van der AH, Ardlie KG, Amos Cl, Criswell LA, Kastner DL, Gregersen PK, Kurreeman FA, Toes RE, Huizinga TW, Seldin MF and Begovich $A B$ : A large scale rheumatoid arthritis genetic study identifies association at chromosome 9q33.2. PLOS Genet 2008, 4: 1000107

2. Plenge R, Padyukov L, Remmers E, Purcell S, Lee A, Karlson E, Wolfe F, Kastner D, Alfredsson L, Altshuler D, Gregersen P, Klareskog $L$ and Rioux J: Replication of putative candidate-gene associations with rheumatoid arthritis in $>4,000$ samples from North America and Sweden: association of susceptibility with PTPN22, CTLA4, and PADI4. Am J Hum Genet 2005, 77: 1044-1060.

3. Plenge R, Seielstad M, Padyukov L, Lee A, Remmers E, Ding B, Liew A, Khalili H, Chandrasekaran A, Davies L, Li W, Tan A, Bonnard C, Liu C, Tian C, Chen W, Carulli J, Beckman E, Altshuler D, Alfredsson L, Criswell L, Amos C, Seldin M, Kastner D, Klareskog $L$ and Gregersen P: REAFI-C5 as a risk locus for rheumatoid arthritis - a genomewide study. $N$ EnglJ Med 2007, 357:1199-1209.

4. Price AL, Paterson NJ, Plenge RM, Weinblatt ME, Shadick NA and Reich D: Principal components analysis corrects for stratification in genome-wide association studies. Nat Genet 2006, 38:904-909.

5. Purcell S, Neale B, Todd-Brown K, Thomas L, Ferreira MAR, Bender D, Maller J, Sklar P, de Bakker PIW, Daly MJ and Sham PC: PLINK: a toolset for whole-genome association and population-based linkage analysis. Am J Hum Genet 2007, 81:559-575.

6. Aulchenko YS, Ripke S, Isaacs A and van Duijn CM: GenABEL: an $\boldsymbol{R}$ package for genome-wide association analysis. Bioinformatics 2007, 23:1294-6.

7. Schemper M: Predictive accuracy and explained variation. Stat Med 2003, 22:2299-308.

8. Wang K, Li M and Bucan M: Pathway-based approaches for analysis of genomewide association studies. Am J Hum Genet 2007, 8I: : 278-1283.

9. GSEA: Gene Set Enrichment Analysis. MSigDB. http://www. broadinstitute.org/gsea/msigdb/collection details.jsp\#C2.

10. Subramanian A, Tamayo P, Mootha VK, Mukherjee S, Ebert BL, Gillette MA, Paulovich A, Pomeroy SL, Golub TR, Lander ES and Mesirov JP: Gene set enrichment analysis: a knowledge-based approach for interpreting genome-wide expression profiles. Proc Natl Acad Sci 2005, 102: I5545-15550.

II. GenMAPP: Gene Map Annotator and Pathway Profiler. http:// www.genmapp.org/.

12. KEGG: Kyoto Encyclopedia of Genes and Genomes. http:// www.genome.jp/kegg/.

13. BioMart. http://www.biomart.org.
Publish with BioMed Central and every scientist can read your work free of charge

"BioMed Central will be the most significant development for disseminating the results of biomedical research in our lifetime. "

Sir Paul Nurse, Cancer Research UK

Your research papers will be:

- available free of charge to the entire biomedical community

- peer reviewed and published immediately upon acceptance

- cited in PubMed and archived on PubMed Central

- yours - you keep the copyright
BioMedcentral 